\title{
An empirical view of knowledge management
}

\begin{abstract}
This chapter is a comprehensive investigative documentary on knowledge management (KM). It was extensively cover past researches done on knowledge management, exposing its varied dimensions to readers as well as guide the readers through its role in research, business, and daily life. The chapter was well discussed about knowledge, knowledge management and knowledge management systems. It also address the Nonaka's Knowledge Management Model or known as SECI modal in order the readers can understand the knowledge creation process.
\end{abstract}

